\title{
Gravitational Driving: Geodesics Warped by Solitons of Acceleration
}

\author{
Nilo Serpa \\ Université des Sciences de l'Homme de Paris - ULSHP, France \\ Centro Universitário ICESP, Brazil \\ nilo.serpa@icesp.edu.br
}

Received: 11 Dec 2020 / Accepted: 04 Jan 2021 / Published: 21 Mar 2021.

\begin{abstract}
This article discusses the basic science to be considered as the background for a speculative technology of gravitational propulsion. Generalized functions are applied to the formalism of general relativity to introduce finite dynamic intervals of spacetime to be warped by acceleration solitons, the latter being interpreted as causing geodesic bends in the spacetime continuum.
\end{abstract}

Key words: soliton, propultion, gravitational tail, warp-drive spacetime, spacetime interval.

Dieser Artikel beschreibt die Grundlagenforschung, die als Hintergrund einer spekulativen Technologie des Gravitationsantriebs betrachtet werden soll. Verallgemeinerte Funktionen werden auf den Formalismus der allgemeinen Relativitätstheorie angewendet, um die richtigen Abstände als dynamische Raumzeitintervalle zu definieren. Einsame Beschleunigungswellen werden so interpretiert, dass sie die geodätischen Abweichungen im Raumzeitkontinuum verursachen.

Schlüsselwörter: Soliton, Antrieb, Gravitationsschwanz, Warp-DriveRaumzeit, Raumzeitintervall.

\section{Prolog}

Physicists (those who do physics, not just teach physics) are usually quite creative. However, judging by the ever-present disagreement as to the interpretation

CALIBRE - Revista Brasiliense de Engenharia e Física Aplicada

Copyright \& 2021, Centro Universitário ICESP. 
of quantum mechanics, and, given the fact that the outcomes of quantum experiments agree extraordinarily with theory, whatever interpretation is given to those outcomes - especially after the irrefutable result of the violation of Bell's inequalities shown by Aspect and his team in the early 1980s - , most physicists repress the contribution of philosophy to understanding reality underlying the experiment. From the point of view of full science practice, I think this is largely unsatisfactory. Here, it is not a question of returning to the old realism along the lines of the discussion between Einstein and Bohr; yes, there must be a realistic model that expresses new relationships between objects in the physical world, a model that allows an objective interpretation, whether stochastic or not, but independent of the subject and the measuring apparatus (not least because there are no variables of the subject in quantum mechanics equations!). Furthermore, mental conditioning to the constructs derived from the first intuition of classical physics and our general perception of the world invariably lead to language problems that, in turn, lead to confused ideas in quantum mechanics.

The inescapable force of geometry on constructive thinking has become so remarkable since immemorial time that it deserved a philosophical exhortation from Pascal:

"Geometry [...] explained the art of discovering unknown truths; it is what it calls 'analysis', and it would be useless to talk about it, after so many excellent works that have been produced"[1]. (author's free translation).

That force undoubtedly characterizes our ways of successfully representing the most immediate reality, at least with regard to survival and exploratory interaction with the world, but it also imprisons us in a thought mold that is difficult to break. In a recent work, I tried to show that, although we create representations inserted in a Euclidean world, nothing prevents other types of relationships being established between objects in domains that are distant from intuition. Quantum entanglement can be an example of fact to be treated under somewhat different spacetime relations. It is not difficult for me to imagine a transformation of symmetry between space and time in such a way that the three spatial coordinates are converted into three time coordinates and the time coordinate is converted into spatial coordinate, configuring new relations in which two entangled particles interact with free transit of information over time (the three time dimensions: past, present and future) along a restricted one-dimensional space connection like an umbilical cord [11].

At the other extreme of reality, space, time and gravity are one and the same thing. In the future, if we are still here and if humanity is freed from its current meanness, when thinking about interstellar travel, we shall need to consider an effective form of space navigation in which spacetime itself propels the vehicle, and not conventional explosions or giant solar sails. In other words, it is no longer a matter of reproducing the concept of daily transport with vessels following point-by-point along a trajectory traced in a void merely receptive to the passage of errant objects. Spaceships shall have to be designed as part of spacetime itself 
and not as something external to it. If the technology for such an achievement shall be attainable, only time can tell us. As for me, I prefer to think like Goethe:

The infinite cannot be said to have parts. All limited existences are in it, not as parts of it, but as infinity participants (free author's translation)[2].

Meanwhile, to go further, it is necessary to break old paradigms, albeit at first conjecturally. This will only be possible through the day-to-day application of philosophy to physicist's work. That is the spirit of this article.

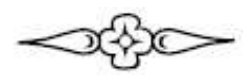

\section{Introduction}

Space travel is one of the most challenging topics for contemporary science. Even with all investments to conceive innovative and sophisticated projects, a physical and intellectual barrier is placed before us: on the one hand, our technology remains fundamentally ballistic, that is, based on projectiles propelled by ignition on conventional fuels; on the other hand, we are stuck with the idea that we must navigate in space, replicating our earthly reality. In a way, the first barrier is conditioned by the second, because/ the mental model of human transport presupposes a displacement in space through an impulse. However, this may not be the only way to understand the transport of matter across the heavens.

Although it is not my goal to speculate on possible interstellar transport technologies, it is worth remembering just one milestone for its innovative theoretical content. I refer to the Alcubierre propulsion model. Basically, the Mexican theoretical physicist Miguel Alcubierre inverted the logic of calculation by using Einstein's equations to determine what type of star would compress space in one direction, expanding it in the opposite direction[4]. Obtaining a relatively simple solution, Alcubierre's propulsion, as it became known, would occur according to a spherical "warp bubble" (containing the starship), hollow of matter and energy, disconnected from outer spacetime, compressing it ahead and expanding it back.

The Alcubierre propulsion model is obtained from a classical-relativistic solution given by an asymptotically-flat warp-drive spacetime metric describing the spherical warp bubble, say

$$
d s^{2}=-c^{2} d t^{2}+\left(d x-f\left(r_{s}\right) v_{s} d t\right)^{2}+d y^{2}+d z^{2},
$$

with a shape function $f\left(r_{s}\right)$ that defines the size and profile of the warp bubble, and where $r_{s}=\sqrt{\left(x-v_{s} t\right)^{2}+y^{2}+z^{2}}$. What emerges from this model is that 
it actually enables timelike observers to travel at superluminal speeds, albeit at the cost of some fictional material with negative rest-mass energy. The bubble moves along the $x$-axis with an arbitrary velocity $v_{s}$ (larger or smaller than the speed of light c).

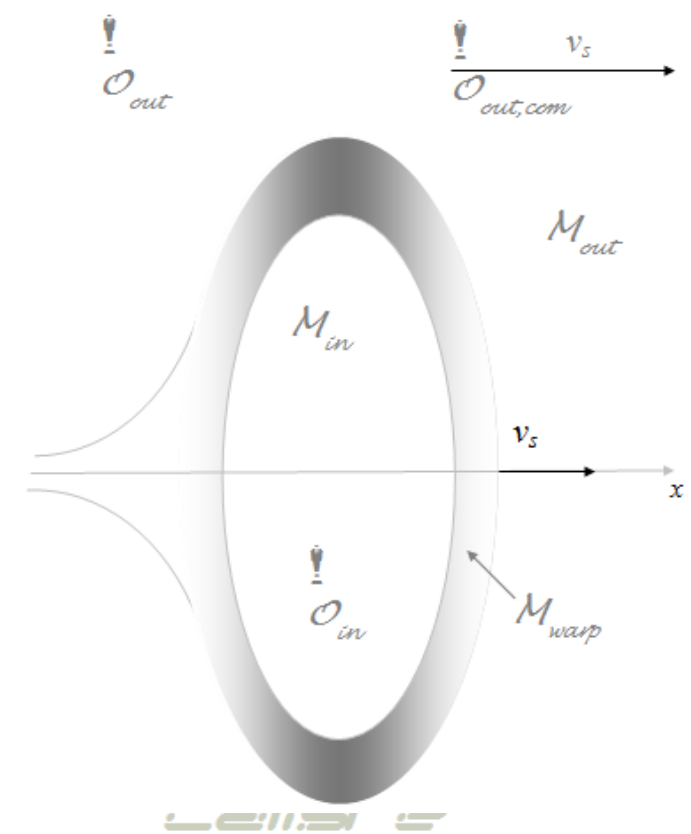

Fig. 1: A representation of the warp-drive spacetime.

Given the above, covering the existing warp-drive spacetimes, we have the general definition of an asymptotically flat vacuum background $\mathcal{M}_{\text {out }}$ enclosing a compact curved manifold with spherical topology (the warping zone $\mathcal{M}_{\text {warp }}$ ) that in its turn carries the traveler region embedded in as an enveloped flat extended compact manifold $\mathcal{M}_{\text {in }}$ endowed of a trivial topology. In Figure 1 it is considered a warp-drive spacetime scheme with axisymmetric front and rear parts along the direction of motion. The remote comoving observer $\mathcal{O}_{\text {out,com }}$ is moving away with constant three-velocity $v_{s}$ relative to the timelike observer $\mathcal{O}_{\text {out }}$, supposed at rest. This three-velocity represents the velocity of the warped region relative to the remote observer $\mathcal{O}_{\text {out }}$, and may be slower, equal or faster than light speed with respect to the observer, a fact that, according to Bobrick and Martire (2021), corresponds to four-velocity of the comoving observer $\mathcal{O}_{\text {out,com }}$ characterized as timelike, null or spacelike. For all practical purposes, 
the warp- drive spacetime is like a warped shell filled by exotic or ordinary matter that moves with constant velocity in relation to an external timelike observer. In principle, the inner compact manifold is modified by the presence of the shell, which leads to differences in times and lengths measured for the inner observer and some commoving remote observer. In short, as pointed out by Bobrick and Martire (2021), "general warp-drive spacetimes[...]form a continuous family which includes both trivial (flat or nearly-flat) and non-trivial (strongly curved) spacetimes" [5].

The standard tools applied to general warp-drives allow us to provide coordinate-invariant measures for the total energy, say

$$
E=\int_{\mathcal{M}_{\text {warp }}}\left(-g^{00}\right)^{-1} T^{00} \sqrt{-g} d^{3} x_{\text {out }}^{i},
$$

where $\left(-g^{00}\right)^{-1} T^{00}$ is the energy density. Recent proposals, variants of the original Alcubierre model — which is in fact a particular element of a wide set of warp-drive solutions with negative energy densities - suggest the plausibility of warp-drives without the need for the extreme properties of the Alcubierre metric, say the negative energy density and correlated tremendous energy demands, provided that the starship is built with a material of exceedingly high density [5] (so high that, of course, it is out of reach for the known science). Also, still from the seminal works of Alcubierre, Lentz demonstrated the existence of superluminal solitons in general relativity, considering positive-energy geometries with energy and momentum conditions conforming to a plasma without net momentum flux[6]. These solitons would contain a central region with minimal tidal forces in such way that Eulerian observers inside this region would remain stationary in relation to the soliton./As expected, positive-energy solitons appear to require the same energy order of magnitude as the original Alcubierre soliton, such as $E_{t o t} \approx(f e w) \times 10^{-1} M_{\odot} v_{s} / c$ for a soliton of moderate dimensions (radius $R=100 m$ and shell thickness $w=1 m)[6]$.

In spite of all the difficulties involved in these conceptions, they make clear the radical turn that we need to consider in order to advance on the subject .

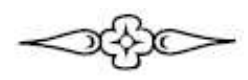

I have devoted much of my scientific disposition to gravity. But as a theoretical physicist and astronomer with technological interests, I have always imagined how the theory could benefit us if we decided to definitely go to the stars (in fact, although it seems the opposite, we are not yet seriously engaged in this; we are busy with trifles, petroleum and how it will be the next cell). Certainly, 
we would have to think differently, conceiving a new way of navigating space, taking advantage of gravity, perhaps. I spent a lot of time thinking about it freely, playing with ideas, some naive, others very serious, until at last I decided to tackle the topic from the simplest things. In particular, two very distinct and very distant images sparked interesting insights during my most recent years of work: one came from the beginnings of quantum mechanics, the old guide-wave model of De Broglie, and the other from the vision of my son, Tane Kanope, surfing on the beaches of Rio de Janeiro. In fact, the general idea is very simple. Navigating gravity means making it accelerate matter. Thus, a material object could, so to speak, "surf" a gravitational warp of a very special type, a finite-energy classical stable solution of conservative non-linear differential field equations in a 4-dimensional space, an acceleration soliton. As in De Broglie's guide-wave, where the particle is guided by the wave crest, the material object is "piloted" by the soliton.

The question connected with such insights is the following: since space, time and gravitation are facets of a unique and indissoluble reality, and, assuming it is possible to develop a technology of gravitational propulsion, what would the geodesics of spacetime look like deformed by the gravitational warp? The answer to this question inevitably leads to the consideration that, in such conditions of movement, matter does not transfer ipsis litteris through the common space but through gravity.

A way of conceiving gravitational propulsion would be to create a queer acceleration wave - a soliton - in the spacetime woof. To create artificially such a soliton we could think of a powerful eletromagnetic field capable of bending geodesic lines according to the shape of the soliton. The material object coupled to the wave must "surf" it following the deformation caused in the geodesics. I do not ignore the difficulties of such an achievement, if possible, starting with the enormous amount of energy needed to create such a deformation in spacetime ${ }^{1}$. To bending the geodesics of spacetime to the point of producing a soliton of the required magnitude is completely out of question in our current technological stage, though we could do some speculations in the direction indicated by Füzfa[3]. In addition, a hypothetical starship itself, built in space with the support of a space station, would have to generate such a field, an unthinkable thing in terms of the existing engineering and energy sources (alternatively, an external device could generate such a field, but there would be the inconvenience of a one-way tour). However, as I said, my aim is not to speculate about the technology that could eventually perform this task, nor to consider space vehicles and all the difficulties involved in a project like this. I just want to imagine what those deformed geodesics would be like, simply establishing a consistent set of basic conjectures ("educated guesses" in the words of Thorne[7]) .

\footnotetext{
${ }^{1}$ According to the equivalence principle, we may generate a gravitational field when generating an electromagnetic field, although the former is very weak compared to the second, so that to make large spacetime deformations it would be needed extremely large electromagnetic fields.
} 
Solitons are non-dissipative objects. They are not exactly oscillations, since these translation waves are solitary "elevations" without corresponding posterior "cavities". As solutions of non-linear equations, solitons propagate along particular directions without changing shape (Figure 2). The non-linearity responsible for the instability of the oscillations is compensated by the interaction with the environment. In the case of a gravitational acceleration soliton, the interaction with the field itself warrants its stability. In any case, non-linearity tends to make the oscillation profile appear more abrupt in the front and slightly softer in the rear, a phenomenon commonly known as "shock wave".

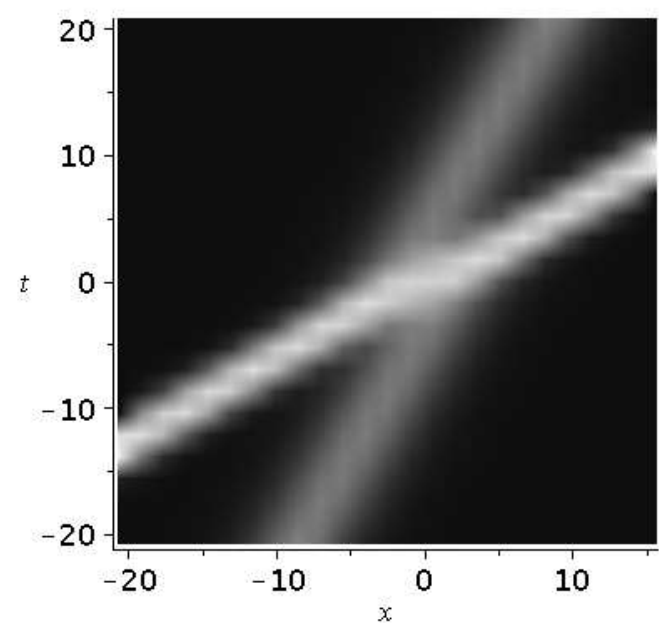

Fig. 2: Interaction between two solitons.

Curiously, the idea of starships guided by solitons has already been the subject of science fiction [8], however, in a superficial way since solitons do not lose energy causing emission of a large amount of radio interference. Of course, on a short time scale, energy dissipation can be disregarded but, on large scales, small energy losses must be taken into account, so that the amplitude of the soliton shall gradually decline, fading at the end. Thus, the usability of the concept shall depend on the intensity of the phenomenon associated with the distance to be overcome in order to control the disastrous effects that could occur from the impact of the soliton on celestial objects. Furthermore, the expected speeds are significant compared to the current ballistics $15 \mathrm{~km}$ per second, but I would be moderate in imagining at first speeds not so dizzyingly close to that of light.

But there is another important aspect ignored by science fiction, which is the natural basis for the applicability of acceleration waves. As in all conjectural work, at least some more universal fundament is needed to support the conjec- 
ture in its initial moments, when it is expected that it can survive the tests of logical consistency and plausibility, insofar as it is known that there is much to be clarified about the world. However, caution is needed. In cosmology, for example, despite the incredible progress of the past 30 years, it is still common to conjecture from a broader conjecture. It is often just what we can do; the problem is how to do it without giving up the physical nexus (not necessarily causal) between both. What I really mean is that the universal fundament I mentioned is the hypothesis of the existence of a conserved acceleration as a remaining "tail" effect from the paleo-gravitational field (the universe as an accelerator since its early stages). In a way, my approach is related to Mach's Principle, according to which the inertial field stems from the entire mass of the universe. For, if gravity and inertia are sides of the same phenomenon, therefore having their origins in mass, and, since we have enough evidence to believe that gravitational manifestations are directly associated with local sources, we can assume that the predominance of inertia at great distances from the local masses can only come from the global mass of the universe, since it would be unacceptable to assume a force that increases with the distance from the local source. Therefore, one can imagine an accelerating universe, since the mass of a body depends on its acceleration in relation to the inertial system (non-local) determined by the rest of the universe.

What follows is conceptually related to the cosmic acceleration induced by the matter of the universe and the propagation of its effects through non-linear deformations in the surroundings of expressive sources of gravitational radiation. Considered the tail effect in the interpretive line mentioned by Bondi [9], the diffusion of those delayed perturbations seems to behave like solitons.

\section{Classical Lagrangian approach: sine-Gordon and Korteweg - de Vries equations}

Solitons are self-sufficient energy solutions of the equations of motion, acting like elementary objects (I prefer not to establish a priori analogies with elementary particles, in order to ensure consistency with my ideas about the elementary complexion of spacetime[10]). I start with a brief reminder of the most wellknown approaches to solitons, paving the way for the theory I will discuss.

So, let me consider a scalar field $\phi$ submitted to Lagrangian formalism:

$$
\frac{\partial \mathcal{L}}{\partial \phi}-\frac{\partial}{\partial t}\left(\frac{\partial \mathcal{L}}{\partial \dot{\phi}}\right)-\frac{\partial}{\partial x}\left(\frac{\partial \mathcal{L}}{\partial \phi^{\prime}}\right)=0,
$$

with

$$
\mathcal{L}=\frac{1}{2}\left[\left(\frac{\partial \phi}{\partial t}\right)^{2}-\left(\frac{\partial \phi}{\partial x}\right)^{2}\right]-m^{2}(1-\cos \phi) .
$$

Substituting Lagrangian density in the former differential equation, we gain the so-called sine-Gordon equation

$$
\frac{\partial^{2} \phi}{\partial t^{2}}-\frac{\partial^{2} \phi}{\partial x^{2}}+m^{2} \sin \phi=0
$$

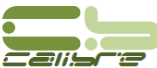


perhaps the most popular equation with a non-linear solitary wave solution. For the system at the lowest energy level (static solution), we must write $-\phi^{\prime \prime}+$ $m^{2} \sin \phi=0$.

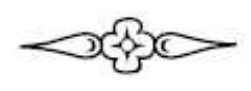

Now, a linear dispersive one-dimensional perturbation propagating along the $\mathrm{x}$-axis would satisfy the equation

$$
\frac{\partial \phi}{\partial t}+\frac{\partial^{3} \phi}{\partial x^{3}}=0
$$

Contrarily, under ñ-linear regime, neglecting the dispersion term $\partial^{3} \phi / \partial x^{3}$, propagation would be governed by

$$
\frac{\partial \phi}{\partial t}+\phi \frac{\partial \phi}{\partial x}=0 .
$$

Thus, a perturbation in which dispersivity is balanced by ñ-linearity would require the presence of both expressions in the equation of motion, something like

$$
\frac{\partial \phi}{\partial t}+\phi \frac{\partial \phi}{\partial x}+\frac{\partial^{3} \phi}{\partial x^{3}}=0 .
$$

Equation (6) is the well known Korteweg - de Vries equation. Going through the assumption

$$
\xi=\dot{x}-w t \Rightarrow \dot{x}=\xi+w t,
$$

we deduce

$$
\begin{gathered}
\partial \dot{x}=\partial \xi ; \\
t=(\dot{x}-\xi) w^{-1} \Rightarrow \partial t=-w^{-1} \partial \xi ; \\
\frac{\partial}{\partial t}=-w \frac{\partial}{\partial \xi} ; \\
\frac{\partial}{\partial \dot{x}}=\frac{\partial}{\partial \xi} .
\end{gathered}
$$

Note that $\dot{x}$ is the generalized coordinate with speed dimension, and $w$ is the acceleration. Since $\partial t=-w^{-1} \partial \xi$, we can return to equation (6) and write

$$
(\phi-w) \frac{\partial \phi}{\partial \xi}+\frac{\partial^{3} \phi}{\partial \xi^{3}}=0 .
$$


Direct integration furnishes

$$
\frac{\partial^{2} \phi}{\partial \xi^{2}}=w \phi-\frac{\phi^{2}}{2}
$$

Multiplying by $\partial \phi / \partial \xi$

$$
\frac{\partial}{\partial \xi} \frac{\partial \phi}{\partial \xi} \frac{\partial \phi}{\partial \xi}=w \phi \frac{\partial \phi}{\partial \xi}-\frac{\phi^{2}}{2} \frac{\partial \phi}{\partial \xi}
$$

and so

$$
\frac{\partial \phi}{\partial \xi} \partial \frac{\partial \phi}{\partial \xi}=w \phi \partial \phi-\frac{\phi^{2}}{2} \partial \phi
$$

Integrating once more,

$$
\begin{gathered}
\frac{1}{2}\left(\frac{\partial \phi}{\partial \xi}\right)^{2}=w \frac{\phi^{2}}{2}-\frac{\phi^{3}}{6} \\
\left(\frac{\partial \phi}{\partial \xi}\right)^{2}=w \phi^{2}-\frac{\phi^{3}}{3}
\end{gathered}
$$

Since hyperbolic second-order partial differential equations encompass wave-like solutions, a perfunctory mathematical analysis allows us to distinguish two diffusion possibilities, namely, 1) all the perturbation travels at once at a given speed and 2) a part of the perturbation is left behind forming a "tail". For the case of the transmission of "gravitational information" associated with loss of mass, Bondi and colleagues were interested in knowing whether gravitational waves obeyed Huygens' principle, or whether tails were produced (in fact, these tails can be obtained from additive terms to the wave equation, or considering the propagating wave in a space of even number of spatial dimensions)[9]. A noteworthy aspect of this investigation was the admission by Bondi that many of the strange features which appeared throughout the work were due more to the method chosen than to the equations themselves (he applied the same treatment to the gravitational wave problem as to the equation of the ordinary scalar wave, recognizing that it was not the simplest way, but the most promising for the case of gravitational waves)[9]. From a heuristic argument on the class of solutions of the wave equation, in particular the class of outward travelling waves, it was possible to generate expressions that tend to zero, which might, in non-linear case, represent tails.

Based on the explanations above, gravitational waves seem to produce tails, that is, parts which fall behind the rest and propagate below the speed of light. Suppose that one of these retarded effects is a specific type of solitary wave, a soliton; as such, it must preserve its shape for a long time, even after a scattering process, until the balance between nonlinearity and dispersivity finally disappears. So, let us assume there is a soliton as a gravitational tail effect reminiscent of the Big-Bang, initially propagating with a speed very close but not equal to $c$. Due to the native ñ-linearity of the early days of the Universe, assuming its age around $4.42 \times 10^{17} s$, and adopting an anisotropic view of the 
cosmic acceleration, we would say that now, since in principle there are no vestiges of such a soliton in the vicinity of the Via-Lactea, $\xi=\dot{x}-w t \approx 0$, while $\partial \phi / \partial \xi$ is fading. Since the gravitational tail is a retarded effect, it can be imagined that differences in the acceleration amplitude over cosmological time would be responsible in part for the differences in acceleration in different regions of the Universe, as the solitary acceleration wave would affect the expansion rate locally.

The problem with this approach is that we once again put ourselves in a privileged position; after all, why would the amplitude of the soliton be zero exactly "here", in Via-Lactea? I do not intend to open this discussion, which should invariably lead to a debate around the anthropic principle. For all practical purposes, in the neighborhood of Via-Lactea, with $\xi \approx 0$ and $\partial \phi / \partial \xi \approx 0$, equation 7 is reduced to

$$
0=w \phi^{2}-\frac{\phi^{3}}{3}
$$

where either $\phi=0$ or $\phi=3 w$.

\section{Spacetime intervals}

From my previous research [10], there are two basic premises for what will follow: the understanding that 1) is the spacetime itself which moves as long as it expands and 2) gravitational attraction between bodies tends to squeeze or compress them as they pull one another toward their center points, which leads to a compression or a squeezing of the spacetime contained between them; a geodesic interval is precisely an element of spacetime under compression or squeezing. An acceleration soliton will necessarily occur in these circumstances, regardless of the intensity of the compression or squeezing.

In my approach, the expression of the invariant commoving element in generalized functions is

$$
d s^{2}=g_{\mu \nu} d\left\langle x_{\mu}-\varepsilon_{\mu}\right\rangle d\left\langle x_{\nu}-\varepsilon_{\nu}\right\rangle,
$$

where $\varepsilon_{\mu}$ and $\varepsilon_{\nu}$ are fixed distances from a point on the spherical boundary of a gravitational manifold called the G-closure. Putting $d\left\langle x_{\mu}-\varepsilon_{\mu}\right\rangle d\left\langle x_{\nu}-\varepsilon_{\nu}\right\rangle=$ $d\langle x-\varepsilon\rangle_{\mu} d\langle x-\varepsilon\rangle_{\nu}$ and applying the variational principle on the commoving element, we get

$$
\begin{gathered}
\delta \int_{A}^{B} d s=\delta \int_{A}^{B} \sqrt{g_{\mu \nu} d\langle x-\varepsilon\rangle_{\mu} d\langle x-\varepsilon\rangle_{\nu}} \delta \int_{A}^{B} \frac{g_{\mu \nu} d\langle x-\varepsilon\rangle_{\mu} d\langle x-\varepsilon\rangle_{\nu}}{\sqrt{g_{\mu \nu} d\langle x-\varepsilon\rangle_{\mu} d\langle x-\varepsilon\rangle_{\nu}}} \\
=\delta \int_{A}^{B} g_{\mu \nu} \frac{d\langle x-\varepsilon\rangle_{\mu}}{d s} \frac{d\langle x-\varepsilon\rangle_{\nu}}{d s} d s .
\end{gathered}
$$

Some manipulations and substitutions lead easily to the first form of the geodesics equation in generalized functions,

$$
\frac{\partial g_{\mu \nu}}{\partial\langle x-\varepsilon\rangle_{k}} \frac{d\langle x-\varepsilon\rangle_{\mu}}{d s} \frac{d\langle x-\varepsilon\rangle_{\nu}}{d s}-2 \frac{d}{d s}\left(g_{\mu k} \frac{d\langle x-\varepsilon\rangle_{\mu}}{d s}\right)=0 .
$$


where the $\langle x-\varepsilon\rangle_{j}$ are compression or squeezing intervals under the rules of Macaulay brakets. One must realize that this formulation in generalized functions aims to focus spacetime intervals, not particles. The introduction of these intervals has the advantage that we can understand spacetime as a geodesic woof in which the arc elements are constantly expanding, or subject to stretching or constriction caused by very massive objects.

\section{Weak effects from gravitational waves}

To generalize the idea discussed in the previous paragraph, I will consider a small perturbation in the spacetime metric, say

$$
g_{\mu \nu}=\eta_{\mu \nu}+h_{\mu \nu},\left|h_{\mu \nu}\right| \ll 1 .
$$

As the targets of the theory are not particles but spacetime intervals, the relevant object to observe the effect of the wave is the spacetime interval itself. Considering the linear regime in the transverse-traceless gauge $(T T)$, we may write

$$
h^{T T}=\eta^{\mu \nu} h_{\mu \nu}^{T T}=0 .
$$

For a time-like vector $t^{\alpha}$

$$
h_{0 \mu}^{T T}=t^{\alpha} h_{\alpha \mu}^{T T}=0 .
$$

Taking into account the freedom of choice of the gauge ${ }^{2}$, it is possible to verify that the TT components of the perturbation are in fact the only ones to satisfy a wave equation in any coordinate system. Therefore, the radiative degrees of freedom of spacetime are represented only by the $T T$ components of the perturbation.

In the absence of a gravitational wave, the spacetime interval is simply

$$
L_{0}=\int\langle x-\varepsilon\rangle_{\mu}^{0} d x=\langle x-\varepsilon\rangle_{\mu}
$$

The passing of a wave determines that

$$
L=\int\langle x-\varepsilon\rangle_{\mu}^{0} \sqrt{g_{x x}} d x=\langle x-\varepsilon\rangle_{\mu} \sqrt{1+h_{x x}^{T T}(t, z=0)} .
$$

Note that the constant of integration is automatically absorbed since it is included in the process, so that, at $x=\varepsilon$, the result will be 0 .

\section{Warped geodesics}

The theoretical basis of the explanation below is well known. Going through the reasoning mentioned above in Section 2, it is now interesting to remember

\footnotetext{
$\overline{2}$ The gauge transformation does not invalidate the linear regime, since it only redefines the perturbation that remains small.
} 
the customary approach, considering a static metric $d s^{2}=-e^{2 \phi\left(x^{1}, x^{2}, x^{3}\right)} d t^{2}+$ $\xi_{i j}\left(x^{1}, x^{2}, x^{3}\right) d x^{i} d x^{j}$ as background for the treatment of null geodesics $\left(d s^{2}=0\right)$. It is worth remembering that time and space are not distinguishable in a dynamic spacetime permeating massive objects such as a binary of black-holes. On the other hand, it is convenient to separate them in a Euclidean manner at the vicinity of planets, for example, where spacetime is static (or almost static); the separation $3+1$ ( 3 components of space and 1 of time) is only the common way we organize objects, how we establish their relationships ${ }^{3}$. This convenience facilitates the approach of the problem, allowing to consider a geodesic of the light-type metric in equivalence to a null-geodesic of the stationary spacetime (static metric $)^{4}$.

Thus, it is useful to define a light-type metric, say $\lambda_{i j}$, which leads to

$$
\tilde{t}^{2}=\lambda_{i j} \tilde{x}^{i} \tilde{x}^{j}
$$

with $x^{i}=x^{i}(s), t=t(s)$, and $\lambda_{i j}=e^{-2 \phi} \xi_{i j}$ (the "tilde" means $d / d s$, while the "wide tilde" means $\partial / \partial s)$. Usually it is tried to prove that a geodesic of the lighttype metric, parameterized by the arc length, is equivalent to a null geodesic of the static metric $\left(e^{2 \phi} \tilde{t}^{2}=\xi_{i j} \tilde{x}^{i} \tilde{x}^{j}\right)$, according to the spatial geodesic equations and the time equation $e^{2 \phi} \tilde{t}=E$.

In fact, I will take this way, however, with two additional assumptions, namely:

1. the variable $x^{i}$ are replaced by $\mathrm{X}^{i}=\langle x-\varepsilon\rangle^{i}$;

2 . the proposed Lagrangian will include the term " $-m^{2}(1-\cos \vartheta)$ ", where $\vartheta=$ $f\left(X^{i}\right)$.

In this study, I will take as basis a sine-Gordon model, although there are others with solitonic properties. In addition, sine-Gordon solitons are especially interesting in physics, including solitons originating in bosonic sine-Gordon theory, which correspond to fermionic states of the Thirring model. However, as I noted earlier, I will not associate solitons with elementary particle states, since the elementary objects in present theory are tiny intervals of spacetime.

General sine-Gordon models are typified by a simple action expression, such as

$$
S_{S G}(\Phi)=\int d^{n} x\left[\frac{1}{2}\left(\partial_{\mu} \Phi\right)^{2}+u \cos (\beta \Phi)\right] .
$$

So, let us take the Lagrangian form

$$
\mathcal{L}=\frac{1}{2}\left(\lambda_{i j} \frac{d \mathrm{X}^{i}}{d t} \frac{d \mathrm{X}^{j}}{d t}\right)-m^{2}(1-\cos \vartheta) .
$$

\footnotetext{
${ }^{3}$ Nothing prevents us from establishing a $1+3$ separation ( 1 component of space and 3 of time), thus describing new relationships between objects ( $\mathrm{I}$ used this to describe quantum entanglement [11]).

${ }^{4}$ By the way, we have a static spacetime if it is stationary and admits a family $\mathcal{M}$ of space-type hypersurfaces orthogonal to its Killing field at any point.
} 
Applying the Euler-Lagrange equation

$$
\frac{d}{d t}\left(\frac{\partial \mathcal{L}}{\partial \dot{X}^{i}}\right)-\frac{\partial \mathcal{L}}{d X^{i}}=0
$$

we have

$$
\begin{gathered}
\frac{\partial \mathcal{L}}{\partial \dot{X}^{i}}=\lambda_{i j} \frac{d \mathrm{X}^{j}}{d t} \\
\frac{\partial \mathcal{L}}{d X^{i}}=\frac{1}{2} \frac{\partial \lambda_{i j}}{\partial \mathrm{X}^{i}} \frac{d \mathrm{X}^{i}}{d t} \frac{d \mathrm{X}^{j}}{d t}-m^{2} \sin \vartheta \frac{\partial \vartheta}{\partial \mathrm{X}^{i}}
\end{gathered}
$$

and so,

$$
\frac{d}{d t}\left(\lambda_{i j} \frac{d \mathrm{X}^{j}}{d t}\right)-\frac{1}{2} \frac{\partial \lambda_{j k}}{\partial \mathrm{X}^{i}} \frac{d \mathrm{X}^{j}}{d t} \frac{d \mathrm{X}^{k}}{d t}+m^{2} \sin \vartheta \frac{\partial \vartheta}{\partial \mathrm{X}^{i}}=0 .
$$

But $\mathrm{X}^{i}(t)=\mathrm{X}^{i}(s(t))$, so that

$$
\frac{d \mathrm{X}^{i}}{d t}=\frac{\partial \mathrm{X}^{i}}{\partial s} \frac{d s}{d t}=\frac{\widetilde{\mathrm{X}}^{i}}{\tilde{t}} .
$$

Combining the last three expressions we gain

$$
\begin{gathered}
\frac{d}{d t}\left(\lambda_{i j} \frac{d \mathrm{X}^{j}}{d t}\right)-\frac{1}{2} \frac{\partial \lambda_{j k}}{\partial \mathrm{X}^{i}} \frac{d \mathrm{X}^{j}}{d t} \frac{d \mathrm{X}^{k}}{d t}+m^{2} \sin \vartheta \frac{\partial \vartheta}{\partial \mathrm{X}^{i}}=0 \\
\frac{d}{d t}\left(e^{-2 \phi} \xi_{i j} \frac{\widetilde{\mathrm{X}}^{j}}{\tilde{t}}\right)-\left(-2 \frac{\partial \phi}{\partial \mathrm{X}^{i}} \xi_{j k}+\frac{\partial \xi_{j k}}{\partial \mathrm{X}^{i}}\right) \frac{\widetilde{\mathrm{X}}^{j} \widetilde{\mathrm{X}}^{k} e^{-2 \phi}}{2 \tilde{t}^{2}}+m^{2} \sin \vartheta \frac{\partial \vartheta}{\partial \mathrm{X}^{i}}=0
\end{gathered}
$$

The fact that $t$ is parameterized by $s, t=t(s)$, with $e^{2 \phi} \tilde{t}=E=$ constant, allows to write

$$
\begin{gathered}
\frac{e^{-2 \phi}}{\tilde{t}} \frac{d}{d t}\left(\xi_{i j} \widetilde{\mathrm{X}}^{j}\right)+\frac{\partial \phi}{\partial \mathrm{X}^{i}} \xi_{j k} \frac{\widetilde{\mathrm{X}}^{j} \widetilde{\mathrm{X}}^{k} e^{-2 \phi}}{\tilde{t}^{2}}-\frac{\partial \xi_{j k}}{\partial \mathrm{X}^{i}} \frac{\widetilde{\mathrm{X}}^{j} \widetilde{\mathrm{X}}^{k} e^{-2 \phi}}{2 \tilde{t}^{2}}+m^{2} \sin \vartheta \frac{\partial \vartheta}{\partial \mathrm{X}^{i}}=0 ; \\
\tilde{t} \frac{d}{d t}\left(\xi_{i j} \widetilde{\mathrm{X}}^{j}\right)+\frac{\partial \phi}{\partial \mathrm{X}^{i}} \xi_{j k} \widetilde{\mathrm{X}}^{j} \widetilde{\mathrm{X}}^{k}-\frac{\partial \xi_{j k}}{\partial \mathrm{X}^{i}} \frac{\widetilde{\mathrm{X}}^{j} \widetilde{\mathrm{X}}^{k}}{2}+m^{2} \widetilde{t}^{2} e^{2 \phi} \sin \vartheta \frac{\partial \vartheta}{\partial \mathrm{X}^{i}}=0 .
\end{gathered}
$$

With the arc length parameterization, we have

$$
\begin{aligned}
& \lambda_{i j} \frac{d \mathrm{X}^{i}}{d t} \frac{d \mathrm{X}^{j}}{d t}=1, \\
& e^{2 \phi}=\xi_{i j} \frac{d \mathrm{X}^{i}}{d t} \frac{d \mathrm{X}^{j}}{d t}, \\
& e^{2 \phi} \tilde{t}^{2}=\xi_{i j} \widetilde{\mathrm{X}}^{i} \widetilde{\mathrm{X}}^{j},
\end{aligned}
$$

which allows writing

$$
\frac{d}{d s}\left(\xi_{i j} \widetilde{\mathrm{X}}^{j}\right)+\frac{\partial \phi}{\partial \mathrm{X}^{i}} e^{2 \phi} \tilde{t}^{2}-\frac{\partial \xi_{j k}}{\partial \mathrm{X}^{i}} \frac{\widetilde{\mathrm{X}}^{j} \widetilde{\mathrm{X}}^{k}}{2}+m^{2} \tilde{t} E \sin \vartheta \frac{\partial \vartheta}{\partial \mathrm{X}^{i}}=0
$$


This expression is the Euler-Lagrange modified sine-Gordon type equation of the geodesic line referring to the metric $d s^{2}=-e^{2 \phi\left(\mathrm{X}^{1}, \mathrm{X}^{2}, \mathrm{X}^{3}\right)} d t^{2}+$ $\xi_{i j}\left(\mathrm{X}^{1}, \mathrm{X}^{2}, \mathrm{X}^{3}\right) d \mathrm{X}^{i} d \mathrm{X}^{j}$, in which an arbitrary interval subject to expansion or contraction is warped by a soliton; the corresponding Lagrangian density is

$$
\mathcal{L}=\frac{1}{2}\left(-e^{2 \phi} \widetilde{t}^{2}+\xi_{i j} \widetilde{\mathrm{X}}^{i} \widetilde{\mathrm{X}}^{j}\right)-m^{2} \tilde{t} E(1-\cos \vartheta)
$$

for

$$
\frac{d}{d s}\left(\frac{\partial \mathcal{L}}{\partial \widetilde{\mathrm{X}}^{i}}\right)-\frac{\partial \mathcal{L}}{\partial \mathrm{X}^{i}}=0
$$

and the arbitrary constant $E$ matches the freedom of the null geodesics affine parameter, being interpreted as the expansion energy contained in the worldline intervals defined at Section 2. In the spatial infinity, admitting that field $\phi$ becomes asymptotically constant, $\lim _{r \rightarrow \infty} \phi=$ const., or that it is cancelled, assuming the limit of small $\vartheta$ we gain

$$
\frac{d}{d s}\left(\xi_{i j} \widetilde{\mathrm{X}}^{j}\right)-\frac{\partial \xi_{j k}}{\partial \mathrm{X}^{i}} \frac{\widetilde{\mathrm{X}}^{j} \widetilde{\mathrm{X}}^{k}}{2}+m^{2} \vartheta \tilde{t} E \frac{\partial \vartheta}{\partial \mathrm{X}^{i}}-\frac{1}{6} \vartheta^{3} \tilde{t} E \frac{\partial \vartheta}{\partial \mathrm{X}^{i}}+\ldots=0,
$$

with remaining terms $\mathcal{O}\left(\vartheta^{5}\right)$ and higher. For $\vartheta=\beta X^{i}$,

$$
\frac{d}{d s}\left(\xi_{i j} \widetilde{\mathrm{X}}^{j}\right)-\frac{\partial \xi_{j k}}{\partial \mathrm{X}^{i}} \frac{\widetilde{\mathrm{X}}^{j} \widetilde{\mathrm{X}}^{k}}{2}+\tilde{t} E \beta^{2} \mathrm{X}^{i}\left(m^{2}-\frac{1}{6} \beta^{2}\left(\mathrm{X}^{i}\right)^{2}+\ldots\right)=0,
$$

and the action is cast in the form

$$
S_{M S G}\left(\mathrm{X}^{i}\right)=\int d^{2} x\left[\frac{1}{2}\left(-e^{2 \varphi} \tilde{t}^{2}+\xi_{i j} \widetilde{\mathrm{X}}^{j} \widetilde{\mathrm{X}}^{k}\right)-m^{2} \tilde{t} E\left(1-\cos \beta \mathrm{X}^{i}\right)\right] .
$$

However, one can think of a more coarse approximation for a very small theta, nearly vanishing the term in $\sin \vartheta$, say

$$
\begin{gathered}
\frac{d}{d s}\left(\xi_{i j} \frac{\partial \mathrm{X}^{j}}{\partial s}\right)=\frac{1}{2} \frac{\partial \xi_{j k}}{\partial \mathrm{X}^{i}} \frac{\partial \mathrm{X}^{j}}{\partial s} \frac{\partial \mathrm{X}^{k}}{\partial s} \\
\frac{\partial}{\partial s}\left(\xi_{i j} \frac{\mathrm{dX}^{j}}{d s}\right)=\frac{1}{2} \frac{\partial \xi_{j k}}{\partial \mathrm{X}^{i}} \frac{\partial \mathrm{X}^{j}}{\partial s} \frac{\partial \mathrm{X}^{k}}{\partial s} ; \\
\partial\left(\xi_{i j} \frac{\mathrm{dX}^{j}}{d s}\right)=\frac{1}{2} \frac{\partial \xi_{j k}}{\partial \mathrm{X}^{i}} \frac{\partial \mathrm{X}^{j}}{\partial s} \frac{\partial \mathrm{X}^{k}}{\partial s} \partial s ; \\
\partial\left(\xi_{i j} \frac{\mathrm{dX}^{j}}{d s}\right)=\frac{1}{2} \frac{\partial \xi_{j k}}{\partial \mathrm{X}^{i}} \frac{\partial \mathrm{X}^{j}}{\partial s} \partial \mathrm{X}^{k}
\end{gathered}
$$

We can integrate this approximation and obtain an expression for the spatial metric from a new parameterization. So, by direct partial integration, we have

$$
\frac{\mathrm{dX}^{j}}{d s}=\frac{\mathrm{X}^{k}}{2 \xi_{i j}} \frac{\partial \xi_{j k}}{\partial \mathrm{X}^{i}} \frac{\partial \mathrm{X}^{j}}{\partial s} \therefore
$$




$$
\begin{gathered}
\frac{\mathrm{X}^{k}}{2 \xi_{i j}} \frac{\partial \xi_{j k}}{\partial \mathrm{X}^{i}}=1 \\
\partial \xi_{j k}=\frac{2 \xi_{i j}}{\mathrm{X}^{k}} \partial \mathrm{X}^{i} .
\end{gathered}
$$

Observing the set of indexes and integrating once more, we write the spatial metric as

$$
\xi_{j k}=2 \xi_{i j} \frac{\mathrm{X}^{i}}{\mathrm{X}^{k}}
$$

Yet, this result has the disadvantage of not containing internal energy $E$ as an impacting factor in the metric relation (in a quantum approach of spacetime, it would be reasonable to imagine that the expansion energy of the geodesic arc interval would play a significant role in this relation). This can be easily solved by resuming equation (27) and repeating the last calculation sequence in the same order, however, previously dividing the entire expression by $E$, so that

$$
\begin{gathered}
\frac{d}{d s}\left(\xi_{i j} \widetilde{\mathrm{X}}^{j}\right)+\frac{\partial \phi}{\partial \mathrm{X}^{i}} E \tilde{t}-\frac{\partial \xi_{j k}}{\partial \mathrm{X}^{i}} \frac{\widetilde{\mathrm{X}}^{j} \widetilde{\mathrm{X}}^{k}}{2}+m^{2} \tilde{t} E \sin \vartheta \frac{\partial \vartheta}{\partial \mathrm{X}^{i}}=0 \\
\frac{1}{E} \frac{d}{d s}\left(\xi_{i j} \widetilde{\mathrm{X}}^{j}\right)+\frac{\partial \phi}{\partial \mathrm{X}^{i}} \tilde{t}-\frac{\partial \xi_{j k}}{\partial \mathrm{X}^{i}} \frac{\widetilde{\mathrm{X}}^{j} \widetilde{\mathrm{X}}^{k}}{2}+m^{2} \tilde{t} \sin \vartheta \frac{\partial \vartheta}{\partial \mathrm{X}^{i}}=0 \\
\frac{E \mathrm{X}^{k}}{2 \xi_{i j}} \frac{\partial \xi_{j k}}{\partial \mathrm{X}^{i}}=1 \therefore \\
=\xi_{j k}=\frac{2 \xi_{i j}}{E^{2}} \frac{\mathrm{X}^{i}}{\mathrm{X}^{k}} .
\end{gathered}
$$

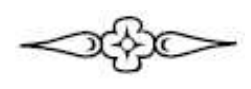

Note that the time variable $t$ is related to the intervals of the spatial metric $\xi_{i j}\left(\mathrm{X}^{1}, \mathrm{X}^{2}, \mathrm{X}^{3}\right)$ considering equation (16). The representation by singularity functions brings, as I suggested earlier, advantages to the understanding, starting with the configuration of real and finite spacetime sections that can be treated as the elementary constituents of the cosmic woof [12] (each spacetime cell very small, however, finite - can be observed under the passage of a soliton; the energy is then transferred to the cells along the worldlines, which could cause local variations in the rate of expansion of the universe). Furthermore, it becomes possible to determine some topological relationships of interest for the physical interpretation of the formalism[12]. 
Certainly, the model expresses a more complex non-linear configuration, presenting greater difficulty in obtaining its soliton-type solutions (in fact, the name soliton is now used in a generalized way, even if the corresponding equation is not integrable in the mathematical sense).

\section{Enhancing the quantum view of spacetime}

Some physicists seem determined to eliminate time from physics. I can think of some reasons for this, but I believe that the main one is the natural human difficulty to get rid of the only type of spacetime relationship that we actually experience, namely, that of common sense. The goal of eliminating time won its advocates, no matter how strange the ideas discussed, or which bring more problems than solutions. Personally, I am skeptical about achieving success with a complete and unified model that is time-free and considers a multiverse; not because of personal preference, but because I still believe in a single universe governed by few general principles and understandable by a relatively simple theory based on a relativistic regime in that the speed of light is held fixed.

If not, let us see a brief argumentation. We can classify the laws of the universe into "ontological laws" — that is, which concern the essence of the phenomena and start from the first principles that are supposed to regulate a single universe (ours) from its origins - , and "methodological laws", which govern experiments carried out at observable scales. It is obviously assumed that the latter are unequivocally connected to the former, otherwise ontological laws would have no relation to the observable world. This is why theories like that of strings are not very productive in this way, since they open a wide range of possible methodological laws: not establishing the first principles translated into initial conditions of the universe, string theory, despite its great beauty, is not able to indicate to us which of its countless solutions (representations of methodological laws) is the one that applies to our universe, giving rise to an infinite number of parallel universes. Under these conditions, we cannot even make unambiguous predictions about anything that is observable in our own universe! I do not consider this a satisfactory alternative, but I hope that string theory, or another equivalent, can shed light on this issue in the near future.

I understand that a creative imagination is essential for physicists, otherwise we wouldn't be able to do physics. However, not everything imagined corresponds to reality. We have lived for some time with the expectation of a satisfactory quantum theory of gravity. For my part, at the moment I prefer to imagine a quantum of spacetime thought in terms of general relativity in an expanding four-dimensional continuum. It is a perfectly fitting hypothesis based on a few principles. I also believe that in four dimensions it is possible to describe the universe as long as we are open to thinking about new four-dimensional relationships between objects. Furthermore, I believe that time is not only real but is the very reason for creation, and that space and time interchange roles in very particular situations. I think that in this line of reasoning it is possible to 
establish a quantization model consistent with general relativity, since what we mean by gravitational field does not carry the same meaning as other fields.

So, if we assume the universe is expanding, we must assume that its elementary constituents are expanding[10]; in a first glimpse of spacetime quantization, we must apply the principle of uncertainty over the product of the interval (similar to position) by its rate of variation due to expansion (similar to speed), in such a way that

$$
\Delta X^{i} \cdot \Delta \widetilde{\mathrm{X}}^{i} \approx \hbar
$$

This approach, however, concerns research that is still ongoing and is not part of the present discussion.

\section{Final remarks and conclusion}

Ambitious mega-projects have been considered among physicists who venture to imagine our future as an interstellar species. Kaku, for example, understands that an anthropic magnetic field around the Martian equator would be possible in a terraforming process, in order to reproduce the cosmic protection that we have on Earth [14]. Basically, it would be necessary, according to Kaku, to build a superconducting network of large magnets surrounding the red planet. It would undoubtedly be an incredible feat. However, a colossal endeavor like this is far beyond the technological capacity of the 21st century. But it is in this line of "educated guesses" that the idea of a solitonic gravitational propulsion is inserted.

The theoretical foundation of mega-projects for generating magnetic fields capable of significantly bending spacetime lies in well-known physics. The EinsteinMaxwell equations for the current loop and solenoid relate the electromagnetic and the gravitational fields by coupling them through the Ricci tensor $R_{\mu \nu}$ and the four-current density $J^{\nu}$ in the system

$$
\left\{\begin{array}{l}
R_{\mu \nu}=-\frac{8 \pi G}{c^{4}} T_{\mu \nu}^{(e m)} \\
\nabla_{\mu} F^{\mu \nu}=\mu_{0} J^{\nu}
\end{array}\right.
$$

where $T_{\mu \nu}^{(e m)}=-\frac{1}{\mu_{0}}\left(g^{\alpha \beta} F_{\mu \alpha} F_{\nu \beta}-\frac{1}{4} g_{\mu \nu} F_{\alpha \beta} F^{\alpha \beta}\right)$ is the Maxwell stress-energy tensor, and $F_{\mu \nu}=\partial_{\mu} A_{\nu}-\partial_{\nu} A_{\mu}$ is the Faraday electromagnetic field tensor. As usual, $g_{\mu \nu}$ is the metric, and $A_{\mu}$ is the four-vector potential. Therefore, the spacetime woof is curved by the energy of the electromagnetic field in accordance with general relativity. From this system, taking into account some additional considerations, Füzfa establishes the dimensionless magneto-gravitational coupling for the current loop and the solenoid [3], say

$$
\begin{gathered}
C_{I}^{\text {loop }}=\frac{8 \pi G}{c^{4}} \mu_{0} I^{2}, \\
C_{I}^{\text {sol }}=\frac{8 \pi G}{c^{4}} \mu_{0} I^{2} n^{2} l^{2} .
\end{gathered}
$$

drawing attention to the fact that it is the square of the total current that sources the gravitational field ( $I$ for the loop and $\operatorname{In} L$ for the solenoid)[3]. By numerical 
resolution of geodesic equations in strongly curved spacetimes around loop and solenoids with extremely large magneto-gravitational coupling, say $C_{I}=10$ or $C_{I}=1$, Füzfa's work culminates in an experimental procedure showing that the effect of generation of artificial gravitational fields with electric currents, although very weak, could be detected through the induced change in spacetime geometry, resulting in classical deflexion of light rays by magnetic fields.

As one can see, there is a consistent theoretical basis in evolution and, in principle, no exotic physics is required. The biggest challenges shall be technological, given the incredible amounts of energy involved. I believe that in order to invest heavily in mega-projects of this extent, we shall need nothing less than constituting a Type II civilization in the Kardashev scale, something that sounds very distant and unlikely given the uncertain chances of our perpetuation (for a rigorous analysis of this issue, see reference [15]). Judging by the absence of vestiges of extraterrestrial life forms technologically capable of interstellar travel, we have the obligation to at least consider the hypothesis that perhaps nature "wisely" has imposed insurmountable physical rules so that possible civilizations never meet, and never take a chance to destroy each other.

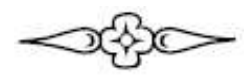

In short, the intent of this article was to show some possible representation for a geodesic deformed by a soliton in the spacetime continuum. Such a soliton would produce a gravitational warp, and would be generated, theoretically, by a powerful electromagnetic field of anthropic origin. In theory, an object with mass could be propelled by this soliton at considerable fractions of light speed. In a broader research program, the next step shall be to deepen the study of that field and its technical features to generate a significant solitonic warp on the geodesics, even as to analyze the potential implications of these ideas on more than forty models of electromagnetism existing today. What is important in the current stage of knowledge is the discussion of theoretical possibilities that may support technological efforts in the future, in my view, still far away.

\section{Acknowledgements}

I would like to thank Prof. Dr. Aparecido Pimentel, Pro-Rector of Research and Innovation at Centro Universitário ICESP. 


\section{References}

1. Pascal, B. (1656/2006). Do espírito geométrico e outros textos — Pensamentos. São Paulo: Escala, 109p.

2. Goethe, J. (1632-1677). Estudo segundo Spinoza. In: Jacira Cardoso (Org.), "Ensaios científicos: uma metodologia para o estudo da natureza", São Paulo: Barany, 116p.

3. Füzfa, A. (2015) "How current loops and solenoids curve space-time" arXiv:1504.00333v3.

4. Alcubierre, M. (1994) "The warp drive: hyper-fast travel within general relativity" Classical and Quantum Gravity 11(5), 73-77.

5. Bobrick, A., Martire, G. (2021) "Introducing physical warp drives" arXiv: $2102.06824 \mathrm{v} 2$.

6. Lentz, E. (2021) "Breaking the warp barrier: Hyper-fast solitons in EinsteinMaxwell-plasma theory" arXiv:2006.07125v2.

7. Thorne, K. (2014). The science of interestellar. New York: W.W. Norton \& Company, 324p.

8. Krauss, L. (1997). A física de Jornada nas Estrelas. São Paulo: Makron Books, 151p.

9. Bondi, H. (1967/1997). Conjetura e mito na física. Brasília: Editora Universidade de Brasília, 57p.

10. Serpa, N., Steiner, J. (2016) "General relativity, quantum gravity and all that: Time machines in perspective by singularity functions" Bulg. J. Phys. 43(1) 1-20.

11. Serpa, N. (2019). "On Wick-rotations and quaternions: The game of symmetry between space and time" CALIBRE 4(2) 1-11.

12. Serpa, N., Fernandes, G. (2020) "The way of entropy: From Lagrangian modelling to thermal engineering" CALIBRE 5(Dec) 1-16.

13. Blanchet, L., Le Tiec, A. (2017) "Tails of gravitational waves and mechanics of compact binaries" https://cqgplus.com/2017/10/09/tails-of-gravitational-wavesand-mechanics-of-compact-binaries/, accessed 12 Jan 2021.

14. Kaku, M. (2018). O futuro da humanidade. São Paulo: Planeta do Brasil, 367p.

15. Serpa, N. (2019). "Fermi's Paradox and Restraint Orders: Why does no one respond?" CALIBRE 4(suplemento) 1-17. 